\title{
The Role of Mixing and Nucleosynthesis in Extremely Metal-Poor Stars and Implications for the Chemical Enrichment of the Galaxy Using the SAGA Database
}

Takuma Suda ${ }^{a, b}$, Shimako Yamada, ${ }^{a}$, Yutaka Katsuta, ${ }^{a}$ Yutaka Komiya, ${ }^{c}$, Wako Aoki, ${ }^{c}$ and Masayuki Y. Fujimoto ${ }^{a}$

${ }^{a}$ Hokkaido University, Kita 10 Nishi 8, Kita-ku, Sapporo, 060-0810, Japan

${ }^{b}$ Keele University, Keele, Staffordshire, ST5 5BG, UK

${ }^{c}$ National Observatory of Japan, Osawa 2-21-1, Mitaka, Tokyo, 181-8588, Japan

E-mail: [suda, yamada, kat, fujimoto] dastro1.sci.hokudai.ac.jp [yutaka.komiya,aoki.wako] anao.ac.jp

\begin{abstract}
We discuss the characteristics of known extremely metal-poor (EMP) stars in the Galaxy using the Stellar Abundances for Galactic Archaeology (SAGA) database*. We focus on the characteristics of carbon-enhanced EMP (CEMP) stars and the possible changeover of the initial mass function (IMF) above $[\mathrm{Fe} / \mathrm{H}] \gtrsim-2.5$. This peak in the IMF at high mass $\left(\sim 10 M_{\odot}\right)$ is supported by studies of the statistics of CEMP stars and the chemical enrichment history of the Galactic halo with respect to the resultant metallicity distribution function. An analysis of the carbon-enhanced stars in our sample suggests that the contribution of AGB stars to the total carbon enrichment differs at metallicities above or below $[\mathrm{Fe} / \mathrm{H}] \sim-2.5$. The transition at $[\mathrm{Fe} / \mathrm{H}] \sim-2.5$ can be interpreted as being due to a change in the nucleosynthesis processes in EMP stars, where at metallicities lower than $[\mathrm{Fe} / \mathrm{H}] \sim-2.5$ hydrogen ingestion into the helium flash convective zones is more prevalent or there is a decline in the ${ }^{13} \mathrm{C}$ pocket efficiency. A statistical test was performed to determine the $\mathrm{Zn}$ and $\mathrm{Ba}$ abundance trends as a function of metallicity. A subsequent Welch's test revealed two abundance breaks at $[\mathrm{Fe} / \mathrm{H}] \sim-2.8$ and -1.9 . The former break corresponds either to the contribution of different supernovae or to the detection limit of spectral lines. The latter one can be interpreted as a result of the changeover of the IMF from a $10 M_{\odot}$ peaked IMF to a Salpeter type $\mathrm{IMF}$ at $[\mathrm{Fe} / \mathrm{H}] \sim-1.9$.
\end{abstract}

PACS: $97.10 . \mathrm{CV}, 97.10 . \mathrm{Tk}, 98.35 . \mathrm{Bd}, 98.35 . \mathrm{Gi}$

11th Symposium on Nuclei in the Cosmos, NIC XI

July 19-23, 2010

Heidelberg, Germany

\footnotetext{
*http://saga.sci.hokudai.ac.jp/
} 


\section{Introduction}

Extremely metal-poor (EMP) stars in the Galactic halo are useful probes for the formation and evolution of our Galaxy. These stars were born in an iron-poor, or perhaps primordial, gas cloud produced soon after the Big Bang and are considered to retain information on the early epoch of the universe. Thanks to the detailed abundance analyses of metal-poor stars by the HK and HES surveys (e.g., [1, 2]), we have data, including elemental abundances, for thousands of EMP candidates, especially for carbon-enhanced stars at low metallicity (see, e.g., [3]).

One of the most important characteristics of observed EMP stars is the presence of abundance anomalies and star to star variations among stars at $[\mathrm{Fe} / \mathrm{H}] \lesssim-2$. In order to understand comprehensively the origins of various elements and the star formation history of our Galaxy, we developed a database of observed EMP stars by compiling observed abundances and related information from the literature (The SAGA database [4]).

One of the essential ingredients in the formation and evolution of galaxies is the initial mass function (IMF), which plays an important role in star formation history. It is found that the stellar population that left EMP stars currently observed in the Galactic halo has a high-mass IMF with a median mass of $5-20 M_{\odot}$ and these EMP survivors were mostly born as the low-mass members of binaries [5]. Furthermore, the work by [6] demonstrates that the high-mass IMF continues up to $[\mathrm{Fe} / \mathrm{H}] \simeq-2.5$ without significant changes from the metallicity distribution function derived by the HK and HES surveys. This in turn implies that there should be a changeover of the IMF from a high-mass one to a low-mass, Salpeter-type mass function.

In this paper, we analyze the abundances of observed EMP stars using the SAGA database. The present paper explores the origins of the carbon-enhanced stars and the possibility of the changeover of the IMF.

\section{Characteristics of Carbon-Enhanced Stars}

Fig. 1 shows the abundance correlation between neutron-capture elements, which separates the contribution from $s$ - and $r$-process for the origins of these elements. In this paper, we define the subclasses in the sample of the SAGA database following [7]. The criterion for carbon-enhanced stars is defined as $[\mathrm{C} / \mathrm{Fe}] \geq 0.7$. The notations "CEMP" and "Crich" in the figure are defined by the metallicity whose critical value is set at $[\mathrm{Fe} / \mathrm{H}]=-2.5$. The labels "EMP" and "MP" are given below and above this metallicity, respectively. Giants, denoted by "RGB" are constrained by $T_{\text {eff }} \leq 6000$ and $\log g \leq 3.5$. Otherwise, the stars are classified as dwarfs and labelled "MS". As a consequence, we have seven classes depending on the abundances and evolutionary status.

The CEMP-s subclass is defined by the criteria $[\mathrm{Ba} / \mathrm{Fe}] \geq 0.5$ and $[\mathrm{Eu} / \mathrm{Ba}]<-0.2$, which is shown by the line in Fig. 1 The line represents the division between sources of the heavy elements. These stars are thought to be born in binary systems and affected by mass transfer from their intermediate-mass primaries in their AGB phase. In the following discussion, we use these criteria for the enhancement of $s$-process elements, and otherwise, attribute the source of neutron capture elements to $r$-process nucleosynthesis, referring to them as " $s-(r-)$ dominant", respectively. In addition, we define CEMP-nos stars as CEMP stars that are not confirmed as $s$-dominant. 


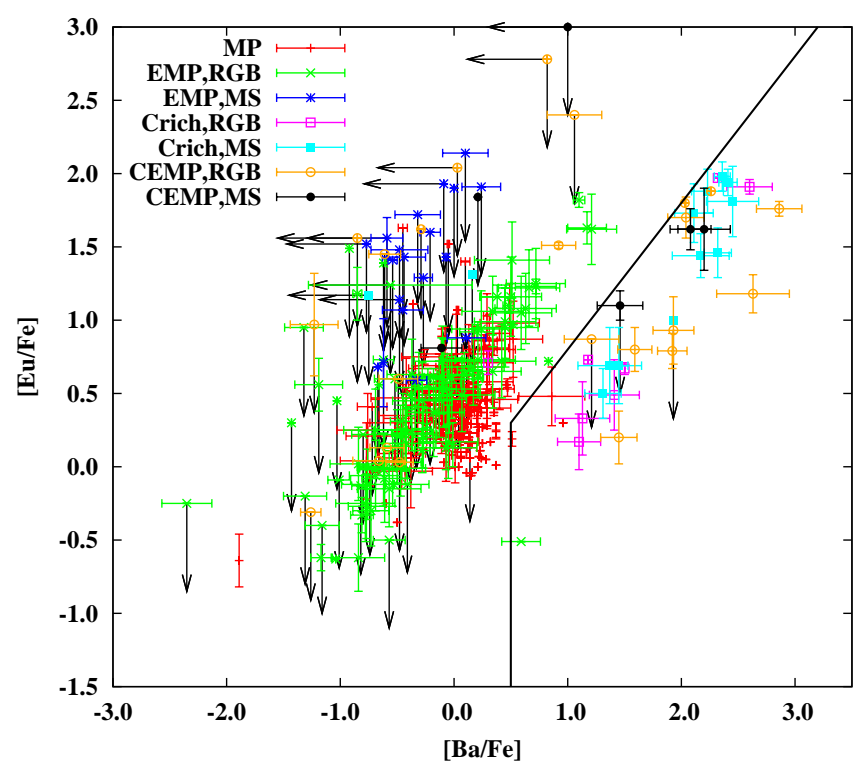

Figure 1: The relation between the $[\mathrm{Ba} / \mathrm{Fe}]$ and $[\mathrm{Eu} / \mathrm{Fe}]$. The production site of heavy elements is separated by a solid line: $[\mathrm{Ba} / \mathrm{Fe}] \geq 0.5$ and $[\mathrm{Eu} / \mathrm{Ba}]<-0.2$. The heavy element abundances plotted to the right of the solid line should come from the $s$-process. The definition of the symbols is given in the text.

We have 365 stars in total in Fig. 1 and 274 of them are classified as $r$-or $s$-process dominant for neutron-capture elements. For MP and EMP RGB groups, almost all the stars are $r$-dominant except for a few stars such as a barium stars HD15096 $([\mathrm{Ba} / \mathrm{Fe}]=0.96)$. The one $s$-dominant "EMP,RGB" star is CS30322-023, which is thought to have originally been a carbon-enhanced star because it shows a large nitrogen enhancement. For the EMP MS group, all Eu abundance data are constrained by upper limits, but should be $r$-dominant because no stars have $[\mathrm{Ba} / \mathrm{Fe}]>0.5$.

Among CEMP stars, 11 out of 21 giants and 3 out of 6 dwarfs are confirmed as $s$-dominant. For C-rich groups, almost all stars (7 out of 8 giants and 12 out of 13 dwarfs) are $s$-dominant for neutron capture elements, whose enhancement of carbon and s-process elements is expected to be caused by the third dredge-up and the ${ }^{13} \mathrm{C}$ pocket formation. Accordingly, $\mathrm{C}$-rich groups have a $s$-dominant origin for neutron-capture elements. This forms a strong contrast with CEMP stars in the sense that the number of CEMP stars almost doubles that of CEMP-nos stars. This is indicative that the $s$-process nucleosynthesis differs in CEMP- $s$ stars from that in the metal-rich counterparts, as argued by [8]; the metallicity range of CEMP stars corresponds to the occurrence of hydrogen mixing into the helium flash convective zones [9,10,11], recalling the argument that CEMP-s stars originate from the He-FDDM event [5] 8, 12].

\section{Changeover of the Initial Mass Function}

As discussed in $\S 1$, the changeover of the IMF is expected at the metallicity of $[\mathrm{Fe} / \mathrm{H}] \gtrsim-2.5$. We explore the possibility of changing the IMF by statistical analyses of abundance trends. Fig. 2 shows the zinc and barium abundances of the sample as a function of metallicity, which reveal abundance breaks at $[\mathrm{Fe} / \mathrm{H}] \approx-2.8$ and $[\mathrm{Fe} / \mathrm{H}] \approx-1.9$. These abundance breaks are based on 

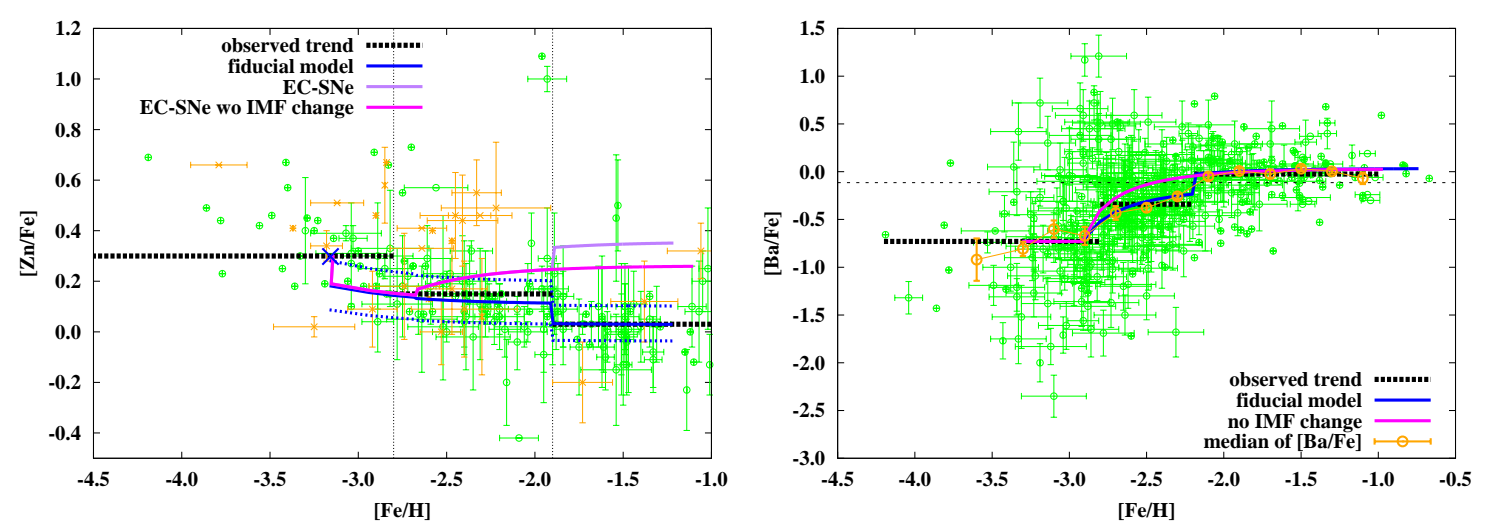

Figure 2: Zinc and barium abundances of the sample as a function of metallicity, with the statistical detection of a possible changeover of the IMF. Solid lines show the chemical evolution models using the IMF peaked at $10 M_{\odot}$ for $[\mathrm{Fe} / \mathrm{H}]<-1.9$ and the Salpeter IMF for $[\mathrm{Fe} / \mathrm{H}]>-1.9$. The IMF average yield from hypernovae is shown by cross for the left-hand panel. The black dotted-line represents the abundance trend inferred from the Welch's test for the mean abundance within specific metallicity bins. Lines with purple and magenta colour are models with electron-capture SNe, with and without the changeover of the IMF at $[\mathrm{Fe} / \mathrm{H}]=-1.9$, respectively. For the left-hand panel, plotted data in orange and green correspond to carbon-enhanced stars and carbon-normal stars, respectively. For the right-hand panel, carbon-enhanced stars are excluded from the sample in order to check the contribution only from the $r$-process.

the Welch's test for the two populations of adjacent metallicity bins from which the data were divided by 0.3 dex each. We set the null hypothesis that the average abundance of the two adjacent metallicity bins do not have the same value. Except for the break metallicities described above, the null hypothesis is rejected at any metallicity bins with $95 \%$ confidence level. We draw flat lines for abundance trends by dashed-lines in the figure except for the breaks, which comes from our assumption that the abundance trends are not changed within the metallicity bins. This assumption holds as long as the Welch's test guarantees that any abundance differences between adjacent populations are within the random error of the abundance.

The first break in the abundance trends can be regarded as the appearance of the contribution of peculiar SNe such as hypernovae associated with the star formation of Pop. III massive stars. The break at this metallicity is not common with other elements probably because the yields of supernova (SN) or hypernovae depend on mass and metallicity. However, it is to be noted that the detection limit of spectral lines to determine the abundances of these elements can affect the trend around this metallicity range.

The second one probably comes from the changeover of the IMF from the $\sim 10 M_{\odot}$ peaked function to a Salpeter-like function. This is because the critical metallicity is found at a similar metallicity for other elements like carbon, oxygen and cobalt (see [7, 13]). This results in the change of yield ratio for zinc by the decreased contribution of HNe and for barium by the increase of contribution of low-mass $\mathrm{SNe}$ where $r$-process elements are expected to synthesize.

We constructed an analytic chemical evolution model that includes the changeover of the IMF at $[\mathrm{Fe} / \mathrm{H}]=-1.9$. Assumptions on models and yields are the same as in [13]; chemical yields averaged over the IMF are metallicity independent and mixed uniformly in the interstellar medium 
to form the next generation stars. Calibration is set at $[\mathrm{X} / \mathrm{Fe}]=0$ when $[\mathrm{Fe} / \mathrm{H}]=0$ in the fiducial model where we start with the log-normal IMF peaked at $10 M_{\odot}$ and replace it with the Salpeter IMF at $[\mathrm{Fe} / \mathrm{H}]=-1.9$. The models well reproduce the abundance trends for both elements. As can be seen in the trend for zinc, electron-capture SNe are not favoured in our model since their contribution becomes significant at $[\mathrm{Fe} / \mathrm{H}] \sim-2.8$.

\section{Acknowledgments}

The authors are grateful to Michael E. Bennett for proof reading the paper. This work has been partially supported by Grant-in-Aid for Scientific Research (18104003), from Japan Society of the Promotion of Science. This work was also supported in part by a grant from the Hayakawa Satio Fund awarded by the Astronomical Society of Japan. T. S. has been supported by a Marie Curie Incoming International Fellowship of the European Community FP7 Program under contract number PIIF-GA-2008-221145.

\section{References}

[1] Beers, T.C. et al. 1992, A search for stars of very low metal abundance. II, ApJ, 103, 1987

[2] Christlieb, N. et al. 2008, The stellar content of the Hamburg/ESO survey. IV. Selection of candidate metal-poor stars, A\&A, 484, 721

[3] Aoki, W. et al. 2007, Carbon-enhanced Metal-poor Stars. I. Chemical Compositions of 26 Stars, ApJ, $\mathbf{6 5 5}, 492$

[4] Suda, T. et al., 2008, Stellar Abundances for the Galactic Archeology (SAGA) Database Compilation of the Characteristics of Known Extremely Metal-Poor Stars, ApJ, 60, 1159

[5] Komiya, Y. et al. 2007, The Origin of Carbon Enhancement and the Initial Mass Function of Extremely Metal-poor Stars in the Galactic Halo, ApJ, 658, 367

[6] Komiya, Y., Suda, T., Fujimoto, M. Y. 2009, Early-Age Evolution of the Milky Way Related by Extremely Metal-Poor Stars, ApJ, 694, 1577

[7] Suda, T. et al. 2011, The Stellar Abundances for Galactic Archaeology (SAGA) Database II Implications for Mixing and Nucleosynthesis in Extremely Metal-Poor Stars and Chemical Enrichment of the Galaxy, MNRAS, 412, 843

[8] Suda, T. et al. 2004, Is HE 0107-5240 A Primordial Star? The Characteristics of Extremely Metal-Poor Carbon-Rich Stars, ApJ, 611, 476

[9] Fujimoto, M. Y., Iben, Jr., I., Hollowell, D. 1990, Helium flashes and hydrogen mixing in low-mass population III stars, ApJ, 349, 580

[10] Fujimoto, M. Y., Ikeda, Y., \& Iben Jr., I. 2000, The Origin of Extremely Metal-poor Carbon Stars and the Search for Population III, ApJ, 529, L25

[11] Suda, T. \& Fujimoto, M. Y. 2010, Evolution of low-and intermediate-mass stars with [Fe/H] $\leq-2.5$, MNRAS, 405, 177

[12] Nishimura, T. et al. 2009, Oxygen and Light-Element Synthesis by Neutron-Capture Reactions in Metal-Free and Extremely Metal-Poor AGB Stars, PASJ, 61, 909

[13] Yamada, S., Suda, T., Komiya, Y., Fujimoto M. Y. 2011, History of Zinc Enrichment and Changeover of Initial Mass Function for Galactic Halo Stars ApJ, submitted 